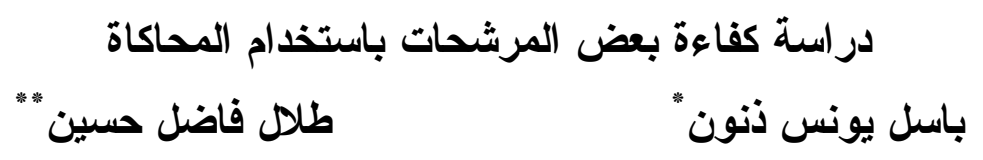

\title{
المستخلص
}

يتضمن هذا البحث در اسة بالمحاكاة لثلاثة مرشحات تستخدم على نطـاق و اسع في النطبيقات الو اقعية. وهذه المرشحات هي مرشـح ألفا-بيتا ومرشح كــالمن

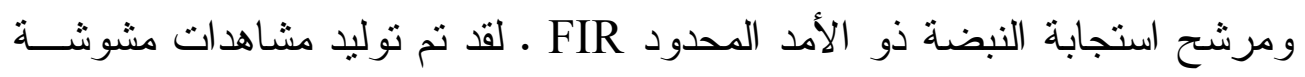
مولدة من ثلاثة نماذج تصادفيه وبالاعتماد على ثلاث عو ائل مختلفة من التوزيعات الاحتمالية مع التشويش. كما اعتمد النطبيق ألحاسوبي الحديث 6 MATLAB في 6 فولى

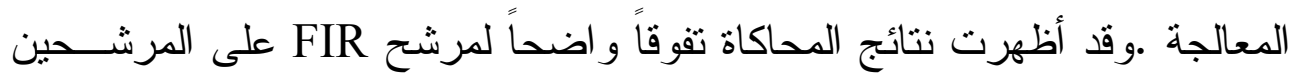
الآخرين.

\section{Studying Consistency of Some Flitters Using Simulation}

\section{ABSTRACT}

This paper includes a simulation study of three well-known filters in real lift application. These filters are $\alpha-\beta$ filter, Kalman filter and FIR filter. Noisily realizations were generated from three stochastic models and depending on three different families of probability distribution of noise. The modern computer application MATLAB 6 is used. The simulation results indicated that FIR filter is more powerful than the other two filters. 


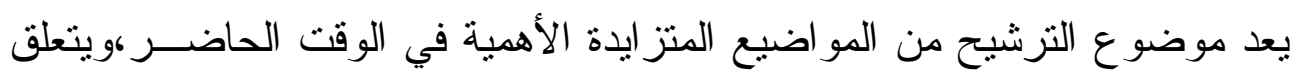

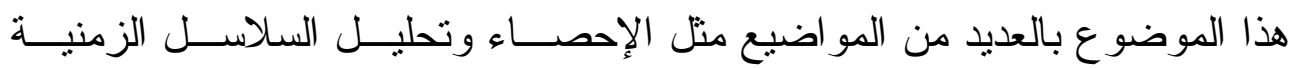

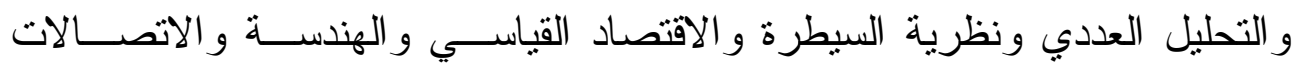

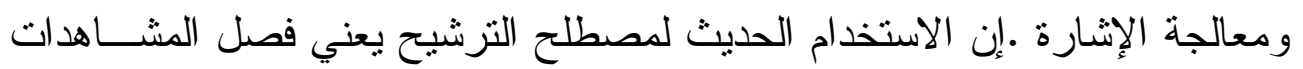

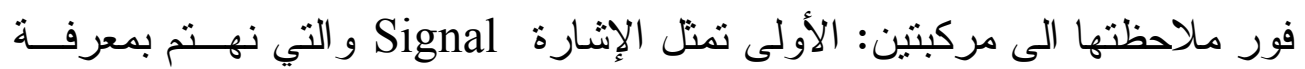
مقدارها، و الثانية هي التشويش Noise و التي تمثل العو امل الطارئســة و المفاجئسـة خارج سيطرتتا. و الثكل الآتي يوضح علية لئي الترشيح.

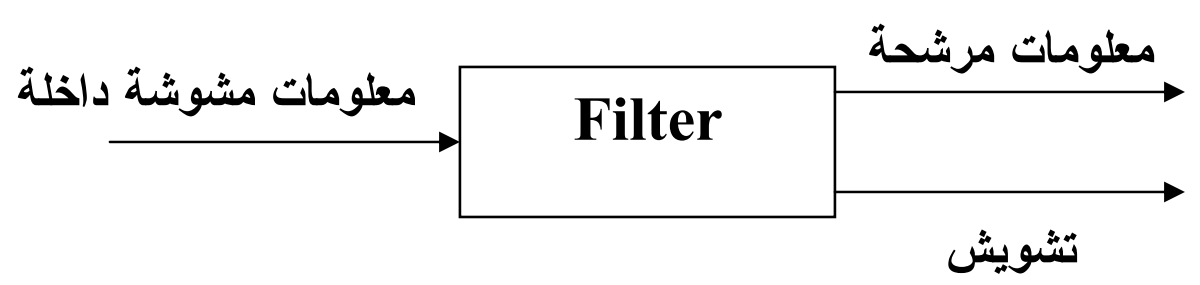

الثكل (1) : عملية الترشيح

إن الاهتمام بموضوع الترشيح يعود إلى العشرينات و الثنلاثينات من القرن العشرين، الا ان هذا الاهتمام زاد منذ ظهور مرشح وينر Wiener Filter الذي صمده العالم

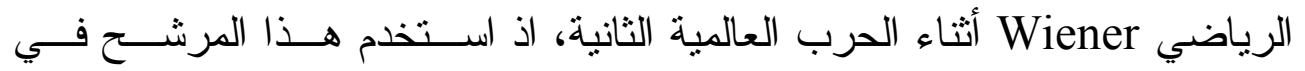
التطبيقات العسكرية و التقنية المختلفة، وفي أو اخــر عــام (1959) وبدايــة عـام

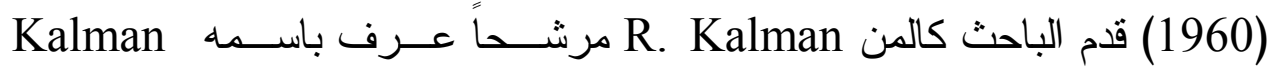

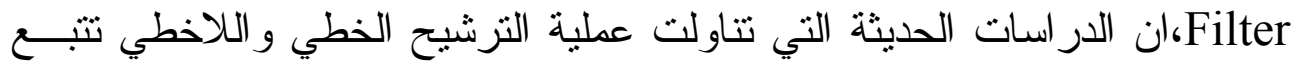

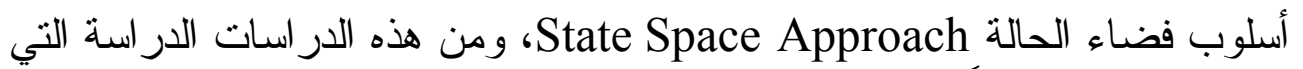
قام بها الباحث (1991) Kitagawa، فقد استخدم أسلوب فضاء الحالة اللاخطـي لـاءي

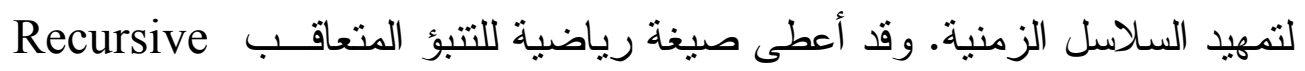

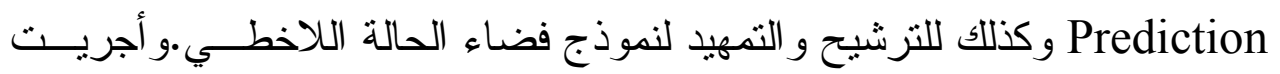

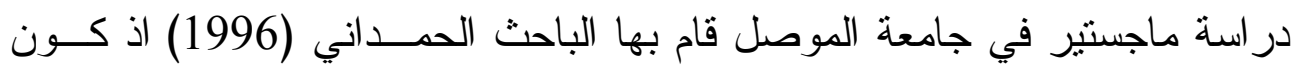

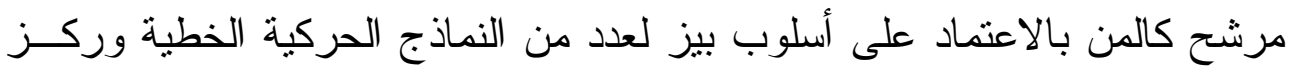

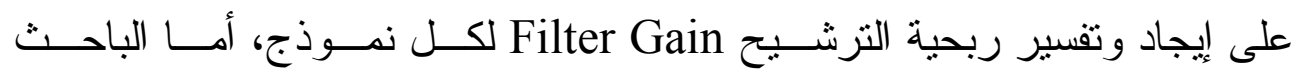


فقد قام بوضع مرشح لاخطــــ Tanazaki (1997)

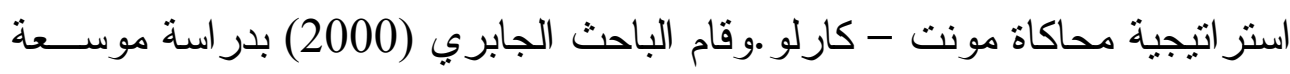

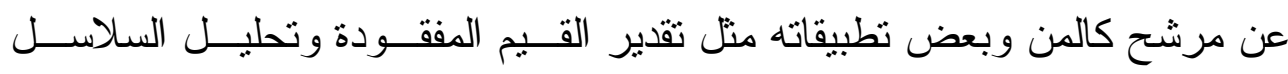

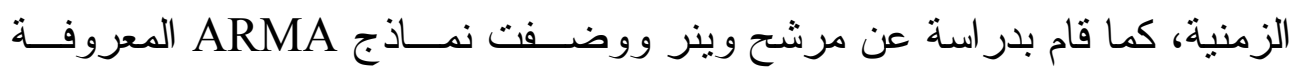

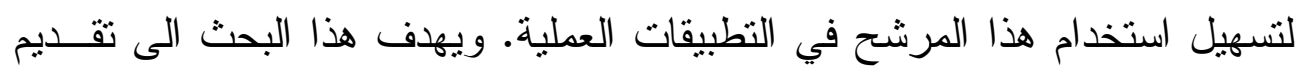

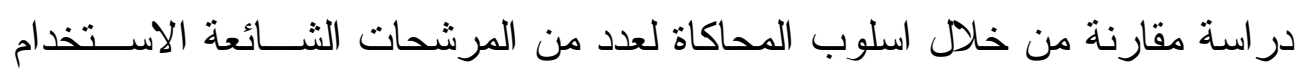

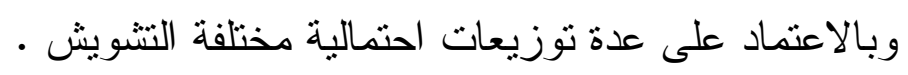

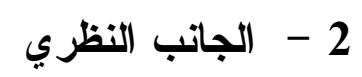

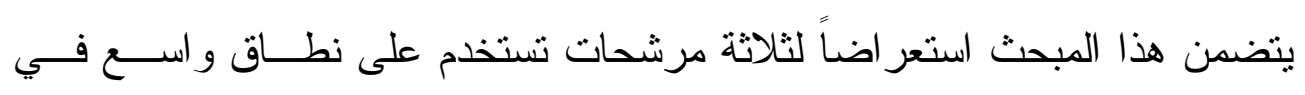

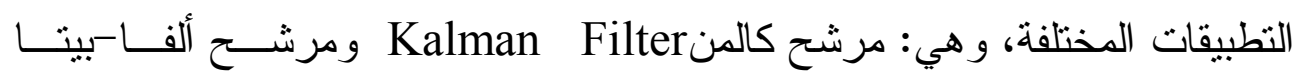

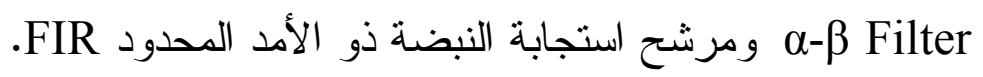
Kalman Filter 1-2 مرشتح كالمن

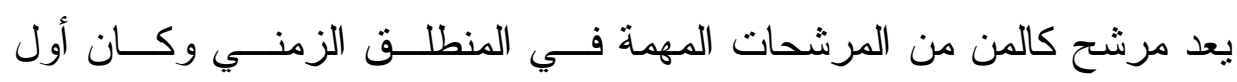

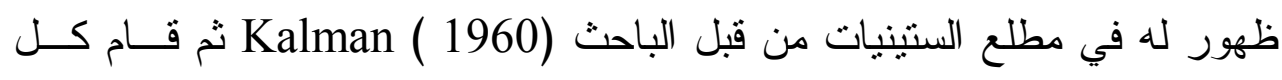

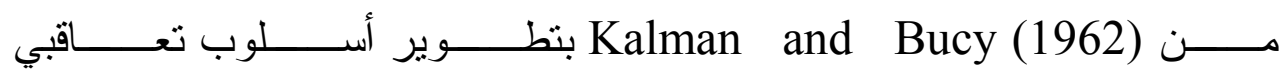
معتمد على تمثيل فضاء الحالة سمي بمرشح كالمن، وقد جاءت فكرة كالمن للتغلب

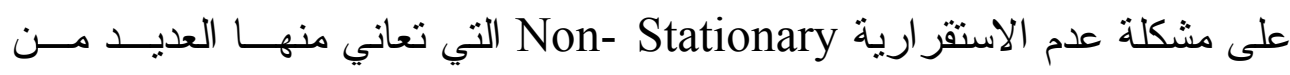
السلاسل الزمنية (الإثارات Signals) الو اقعية.إن أهم ما يميز مرشح كالمن هي لإني

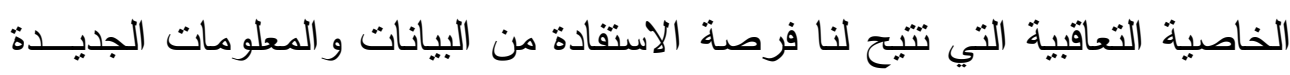

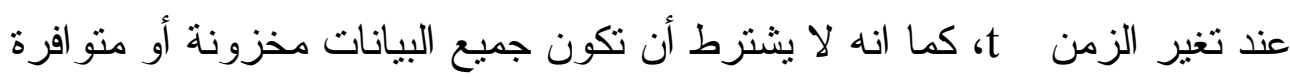

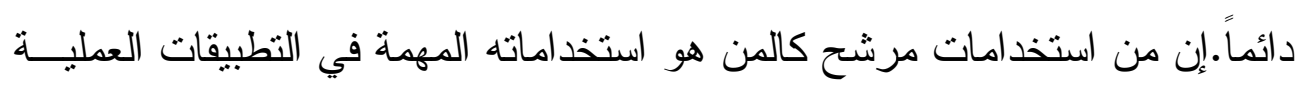

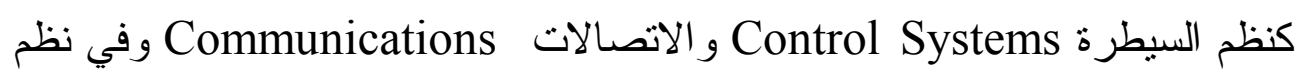
الر ادارات وتعقب الأهداف البحرية و الجوية. وقد توسعت تطبيقاته حتى شملت شتى مجالات الحياة الثقنية و الهندسية و الحاسوبية و الطبية الدختلفة. 
إن مرشح كالمن للنظام الديناميكي يتكون من معادلتين أساسيتين: الأولـى لـى

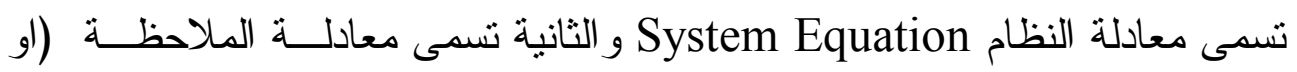
المشاهدة) (Observation Equation ) System Equation: $\quad \mathrm{X}_{\mathrm{t}}=\phi_{\mathrm{t}} \mathrm{X}_{\mathrm{t}-1}+\omega_{\mathrm{t}}$

Observation Equation: $\quad y_{t}=M_{t} X_{t}+v_{t}$

حيث إن ف هو عبارة عن تتشويش النظام System Noise و عادة يكــون

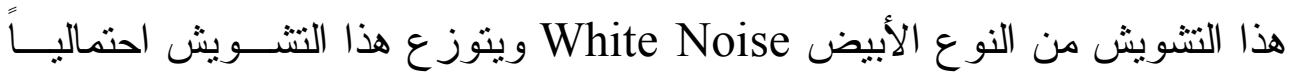
Observation بمعد صفر وتباين

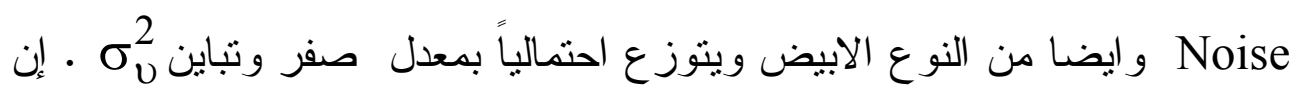

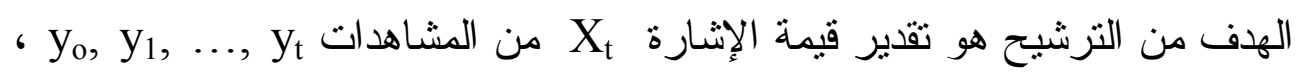

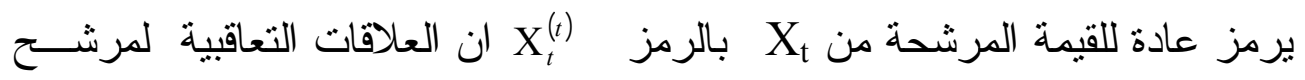

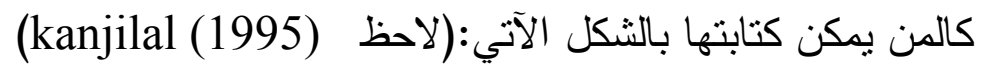
$\mathrm{X}_{\mathrm{t}}^{(\mathrm{t}-1)}=\phi_{\mathrm{t}} \quad \mathrm{X}_{\mathrm{t}-1}^{(\mathrm{t}-1)}$

$\mathrm{P}_{\mathrm{t}}^{(\mathrm{t}-1)}=\phi_{\mathrm{t}} \quad \mathrm{P}_{\mathrm{t}-1}^{(\mathrm{t}-1)} \quad \phi_{\mathrm{t}}^{\prime}+\mathrm{Q}_{\mathrm{t}}$

$\mathrm{k}_{\mathrm{t}}=\mathrm{p}_{\mathrm{t}}^{(\mathrm{t}-1)} \mathrm{M}_{\mathrm{t}}\left(\mathrm{M}_{\mathrm{t}} \mathrm{P}_{\mathrm{t}}^{(\mathrm{t}-1)} \mathrm{M}_{\mathrm{t}}^{\prime}+\mathrm{R}_{\mathrm{t}}\right)^{-1}$

$\mathrm{X}_{\mathrm{t}}^{(\mathrm{t})}=\mathrm{X}_{\mathrm{t}}^{(\mathrm{t}-1)}+\mathrm{k}_{\mathrm{t}}\left(\mathrm{y}_{\mathrm{t}}-\mathrm{M}_{\mathrm{t}} \mathrm{X}_{\mathrm{t}}^{(\mathrm{t}-1)}\right)$

$\mathrm{P}_{\mathrm{t}}^{(\mathrm{t})}=\mathrm{P}_{\mathrm{t}}^{(\mathrm{t}-1)}-\mathrm{K}_{\mathrm{t}} \mathrm{M}_{\mathrm{t}} \mathrm{P}_{\mathrm{t}}^{(\mathrm{t}-1)}$

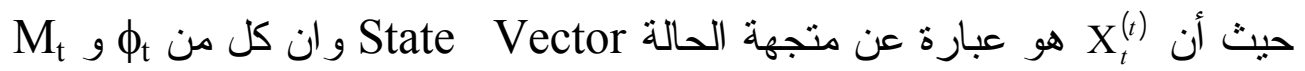

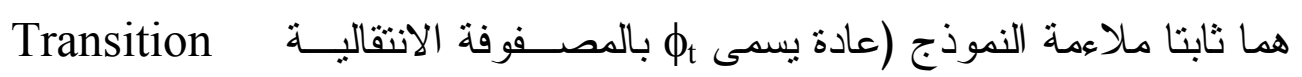
(Transformation Matrix بمصفوفة التحويل Matrix تباين تشويش المشاهدة (و الذي يكافي V

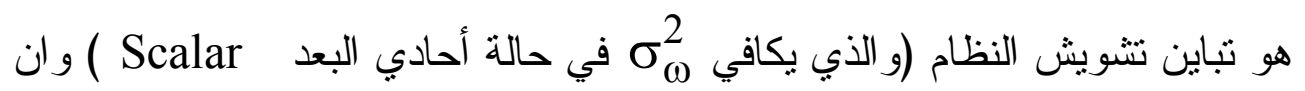

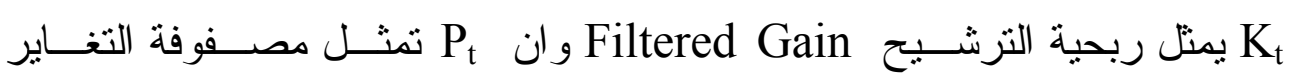
Covariance Matrix

$$
\text { مبينة في الجابري(2000) }
$$




\section{مزايا مرشح كالمن}

يتمتع مرشح كالمن بشهرة واسعة نتيجة للعديد من المز ايا الإيجابية التي يتمتع بهــا ومنها:

أ.انه مرشح بالغ العمومية يمكن استخدامه مع مختلف السلاسل الزمنية: المسـتقرة وغير المستقرة، الخطية وغير الخطية. ب.انه مرشح ذو طبيعة تعاقبية مما يجعله مناسباً للتعامل مع الحاسوب.

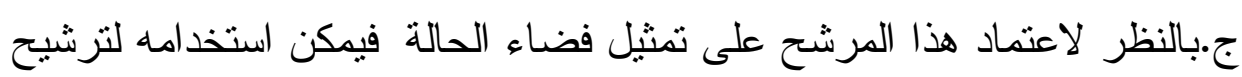
سلاسل زمنية متعددة في آن واحد هي النوازي ( Parallel) د.انه مرشح ذو ربحية ترشيح متغيرة بتغير الزمن مما يؤدي إلى تصحيح المرشح

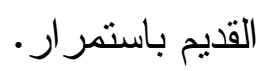
هـ.يمكن لهذا المرشح النرشيح الفوري مما يؤهله للاستخدام مع النظم الحية.

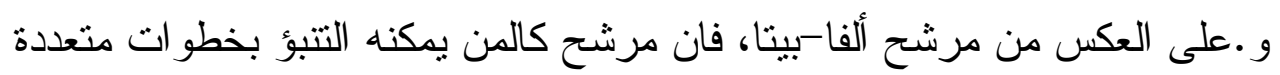

$$
\text { } 2.2 \text { مرشع ألفا - بيتا }
$$

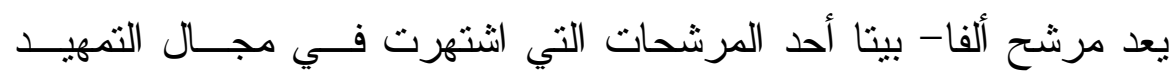

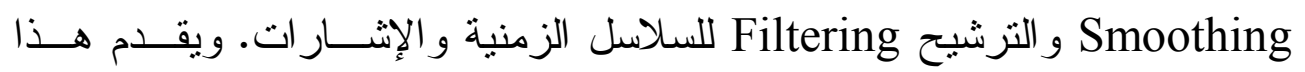

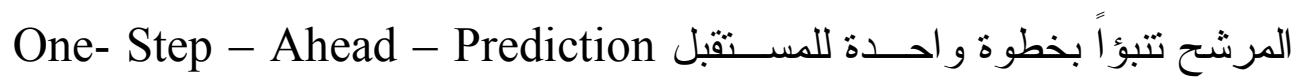

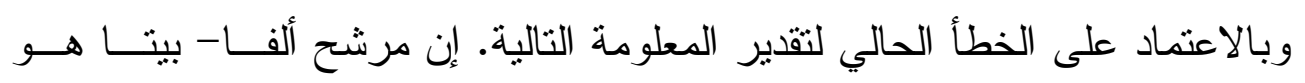

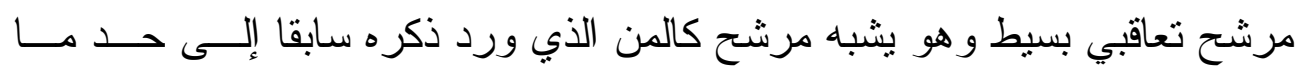

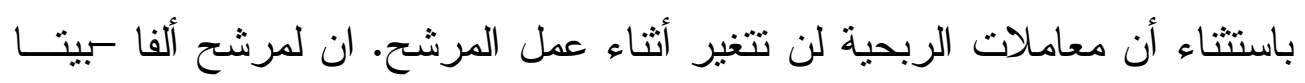

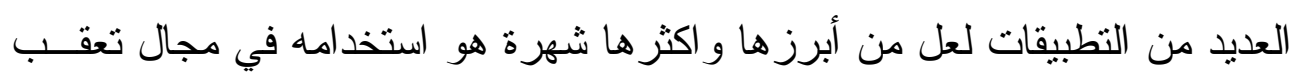

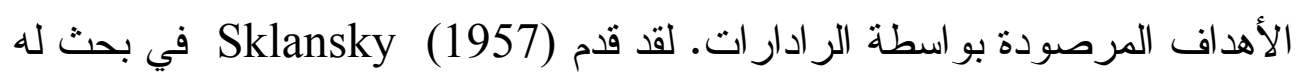
تحليلاً لسلوك مرشح ألفا-بيتا اذ عرض في هذا التحليل المدى المقبول لقيم معلمات

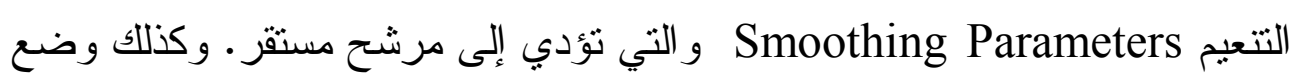
القيود Constraints على هذه المعلمات. كما اثشتق صيغا مغلقة لإيجاد العلاقة بين 
مقدر التتعيم وامكانية الترشيح في حالة كون التشويش ابيض White Noise وقام

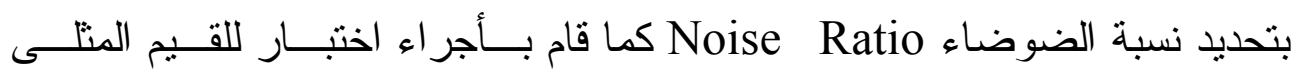
Optimal Values

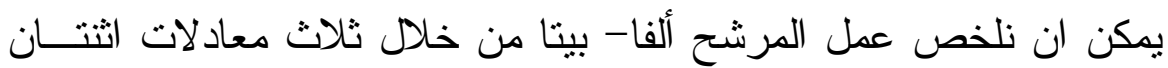
منها للتتعيم والأخيرة للتقدير وبالثكل الاتي و على التو الي:

$$
\begin{aligned}
& X_{f}(t)=X_{p}(t)+\alpha\left[X_{0}(t)-X_{p}(t)\right\rfloor \\
& D_{f}(t)=D_{f}(t-1)+\beta\left[X_{0}(t)-X_{p}(t)\right\rfloor \\
& X_{p}(t+1)=X_{f}(t)+D_{f}(t)
\end{aligned}
$$$$
\text { (Lefferts (1981) (لاحظ) }
$$

حيث إن Observed Value تمثل القيمة الملاحظة Obا للمشاهدة عند الزمن t. القيمة التتبؤية Predicted Value ل للمشاهدة عند الزمن X

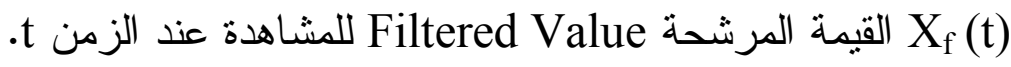
Position Smoothing Parameter هي معلمة تمهيد الموقع المعند

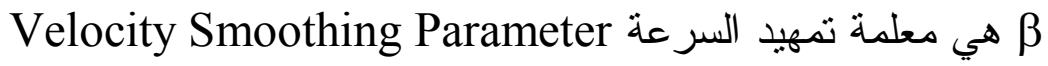
(يقصد بالموقع و السرعة للهدف الجوي - في حالة تعقب الأهداف الجوية).

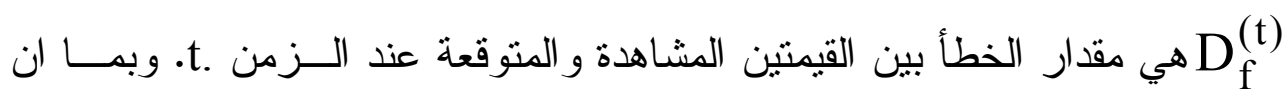
معادلة التوقع قد وردت بصيغة تعاقبية، فهذا يعني أنها تحتاج إلى قيمــة ابتدائيــة. وتحسب القيمة الابتدائية عادة وذلك بأخذ القيمة المتوقعة مساوية للقيمة المشــاهدة مباشرة، كما تؤخذ قيمة D الابتدائية بوصفها الفرق بـين قيمتــين أو مشـــاهدتين

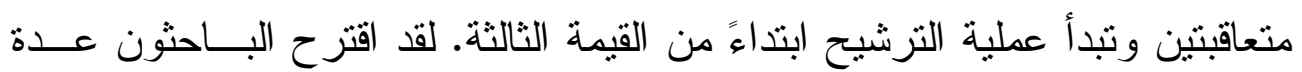

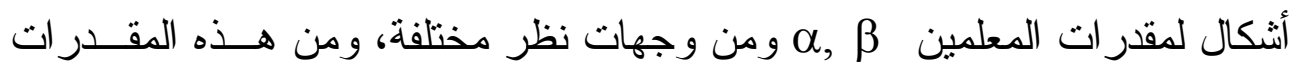

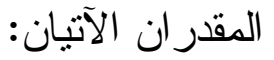




$$
\begin{aligned}
& \hat{\alpha}=\frac{2(2 n-1)}{n(n+1)} \\
& \hat{\beta}=\frac{6}{n(n+1)}
\end{aligned}
$$

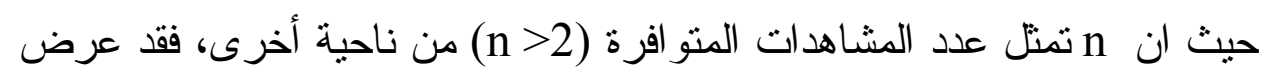

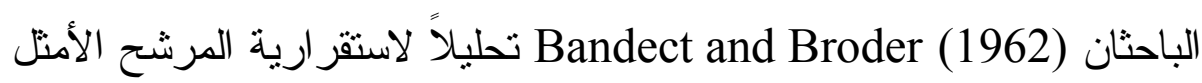
Minimum Cost و الذي يعطي اقل دالة كلفة Optimal Filter Function الضوضاء. وقد وجدا بان المرشح الأمثل يمكن الوصول اليه باختيار مقدر للمعلمة

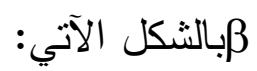

$$
\hat{\beta}=\frac{\alpha^{2}}{2-\alpha}
$$

أما المعلمة م فيمكن اختبار ها ضمن الفترة (0,2). ونشير إلى ان الباحث

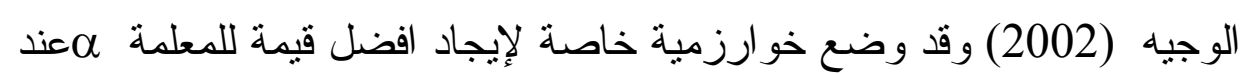
قيمة معينة من $\beta$ كما توصل الى طر ائق اكثر كفاءة لتقدير هاتين المعلمتين.

\section{مزايا مرشح ألفا-بيتا}

يعد مرشح ألفا-بيتا من المرشحات الثائعة في الاستعمال، وخاصة في مجال تعقب

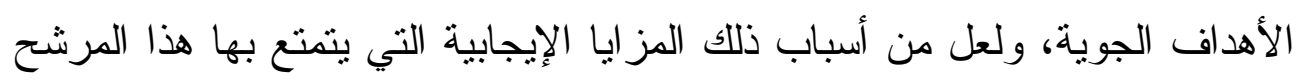
ومنها:

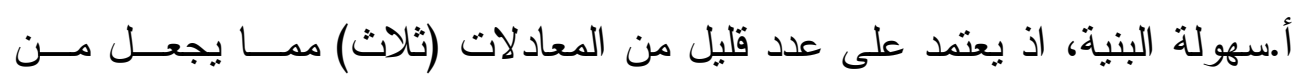

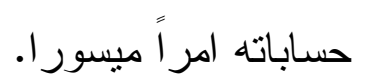

ب.آنية التففذ وسر عة العمل نتيجة لسهولة البنية التركيبية لهذا المرشح. جـققلة البيانات المطلوبة كقيم بدائية (فقط نحتاج إلى قيمتين بدائينتين عند المباثرة بتطبيق هذا المرشح). 


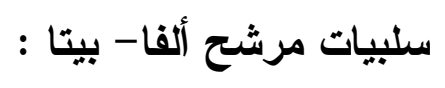

أ.الحاجة إلى اختيار قيم بدائية بدقة جيدة وخبرة عالية لتقدير معلمات التمهيد.

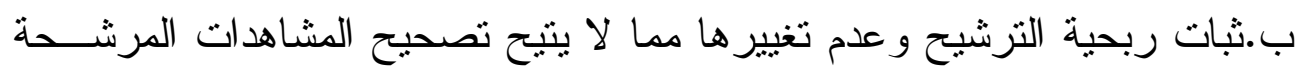

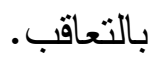
جـ.لا يمكن استخدام هذا المرشح للتنبؤ بأكثر من خطوة واحدة.

\section{2 مرشح استجابة النبضة ذو الأمد المحدود}

\section{Finite-Duration Impulse Response (FIR) Filter.}

يعد هذا المرشح من المرشحات الرقمية الثنائعة الاستعمال في معالجة الإثارة فـي

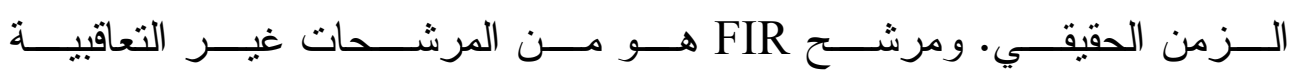
في المنطلق الزمني و الذي يكون له (في حالة النظم السبيية

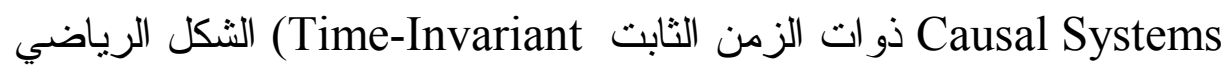
الآتي:(لاحظ (1998) دمن (Carlson)

$$
x(t)=\sum_{i=0}^{q} h(i) y(t-i) \quad ; \quad \mathrm{t}=0,1,2, \ldots
$$

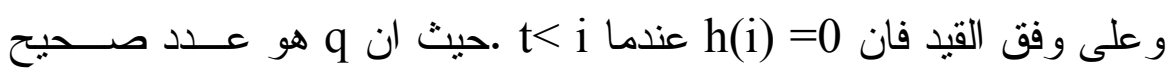

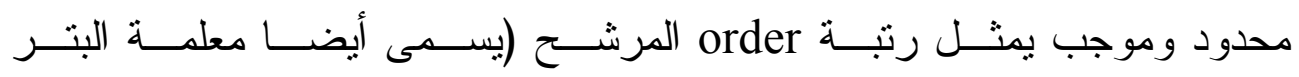

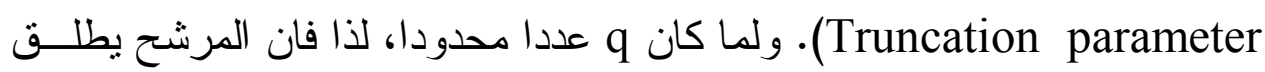

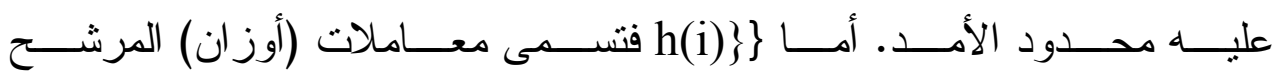
ويمكن الاستتتاج بيسر ان المعادلة (5ilter coefficients (weights) تحويلها بشكل الصيغة الدصفوفية وكما يأتي: 


$$
\begin{aligned}
& {\left[\begin{array}{c}
x(0) \\
x(1) \\
x(2) \\
\cdot \\
\cdot \\
\cdot \\
x(q)
\end{array}\right]=\left[\begin{array}{ccccccc}
h(0) & 0 & 0 & . & \cdot & 0 \\
h(1) & h(0) & 0 & . & \cdot & \cdot & 0 \\
h(2) & h(1) & h(0) & \cdot & \cdot & . & 0 \\
\cdot & \cdot & \cdot & \cdot & \cdot & \cdot & \cdot \\
\cdot & \cdot & \cdot & . & \cdot & \cdot & \cdot \\
\cdot & \cdot & \cdot & . & \cdot & \cdot & \cdot \\
h(q) & h(q-1) & h(q-2) & . & . & . & h(0)
\end{array}\right]\left[\begin{array}{c}
y(0) \\
y(1) \\
y(2) \\
\cdot \\
\cdot \\
y(q)
\end{array}\right]} \\
& \mathbf{x}=\mathrm{H} \mathbf{y} \\
& \mathbf{y}=\left[\begin{array}{lllll}
\mathrm{y}(0) & \mathrm{y}(1) & \mathrm{y}(2) & \ldots \mathrm{y}(\mathrm{q})
\end{array}\right]^{\prime} \\
& \mathbf{x}=\left[\begin{array}{llll}
\mathrm{x}(0) & \mathrm{x}(1) & \mathrm{x}(2) \ldots \mathrm{X}(\mathrm{q})
\end{array}\right]^{\prime} \\
& \mathrm{H}=\left[\begin{array}{ccccccc}
\mathrm{h}(0) & 0 & 0 & . & . & . & 0 \\
\mathrm{~h}(1) & \mathrm{h}(0) & 0 & . & . & . & 0 \\
\mathrm{~h}(2) & \mathrm{h}(1) & \mathrm{h}(0) & . & . & . & 0 \\
\cdot & \cdot & \cdot & . & \cdot & \cdot & . \\
\cdot & \cdot & \cdot & . & \cdot & \cdot & . \\
\cdot & \cdot & . & . & . & . & . \\
\mathrm{h}(\mathrm{q}) & \mathrm{h}(\mathrm{q}-1) & \mathrm{h}(\mathrm{q}-2) & . & . & . & \mathrm{h}(0)
\end{array}\right] \\
& \text { حيث إن }
\end{aligned}
$$




$$
\begin{aligned}
& \text { وبالعودة إلى المعادلة (6a) نلاحـظ ان هنــالك (q+1) مسـن المجاهيـلـ }
\end{aligned}
$$

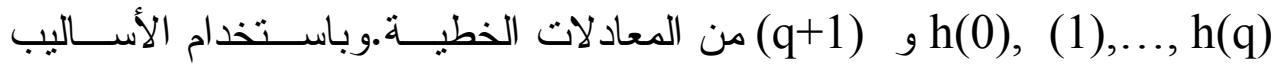

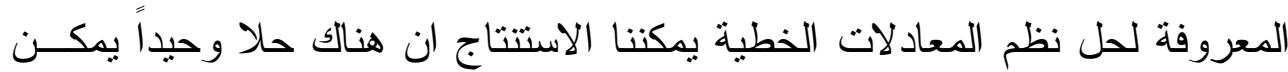

$$
\begin{aligned}
& \text { التعبير عنه من خلال العلاقات التعاقبية الآتية: } \\
& \mathrm{h}(0)=\mathrm{x}(0) / \mathrm{y}(0) \\
& h(i)=\left[x(i)-\sum_{j=0}^{i-1} y(i-j) h(j)\right] / y(0) ; \quad i=1,2, \ldots q
\end{aligned}
$$

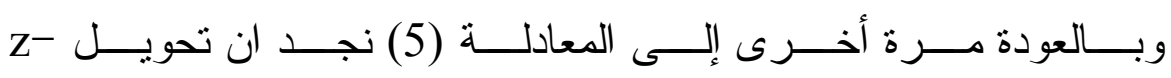

$$
\begin{aligned}
& \text { z-Transform لهذه المعادلة يكون كما يأتي (لاحظ (1998) (Carlson): } \\
& X(z)=\sum_{i=0}^{q} h(i) z^{-i} Y(z)
\end{aligned}
$$

لذا فان دالة تحويل Transfer Function مرشح FIR يكون لها الصيغة الآتية: $H(z)=X(z) / Y(z)=\sum_{i=0}^{q} h(i) z^{-i}$

ان النظام المتمنل بالمعادلة (9b) ليس له تغذية رجعية Feedback وذلك

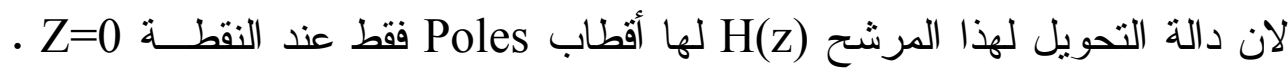

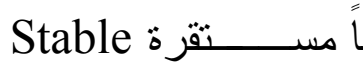
ائم FIR تهات

$$
\text { (انظر (1998)(Carlson) }
$$

\section{FIR Fزايا مرشح}

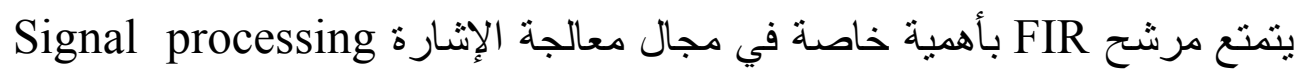
ويمكن إجمال أهم ميزاته بالنقاط آلاتية: أ.ان مرشحات FIR سهلة التمثيل رياضياً، لذا فهي سهلة الاستخدام.

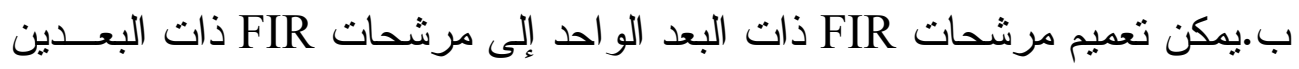

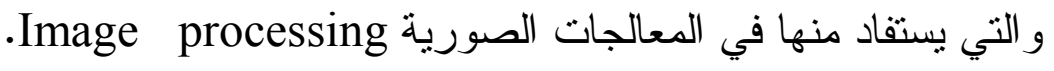

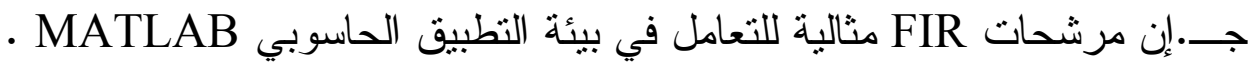

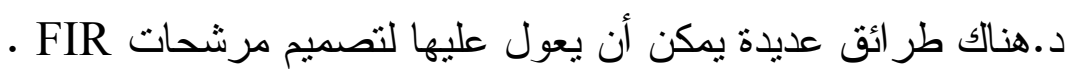
هـ. مرشحات FIR تكون مستقرة Stable دائماً. 
3- اختبار كفاءة المرشحات بالمحاكاة

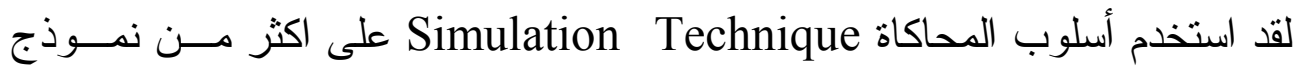
رياضي واحد وباستخدام توزيعات احتمالية مختلفة لغرض اختبار كفاءة المرشحات

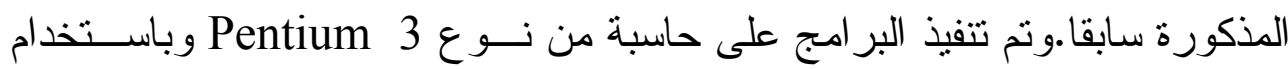
التطبيق الحاسوبي الحديث MATLAB 6 ، لقد اعتمدت ثلاثة نوزيعات احتماليــة فئس مختلفة لغرض توليد التشويش Noise و الذي يرمز له بالرمز Zt و وهذه التوزيعات

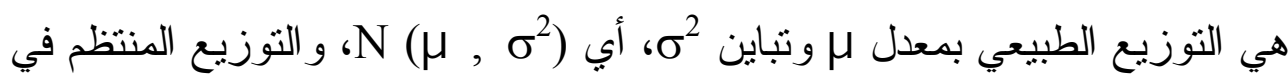

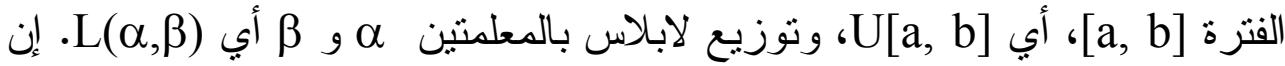

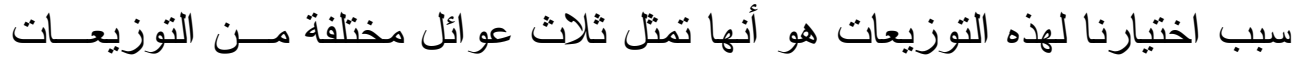

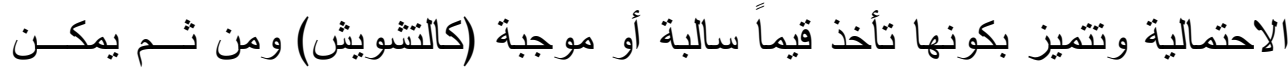
تثبيت الأوساط الحسابية لهذه التوزيعات عند الصفر (كما يفترض في اغلب الأحيان للتشويش). فلو ثبتتا معدل التشويش ليكون ب=0 ولو اعتبرنا أن تباين التشويش هو ( $\sigma^{2}$ يكون لها المعدل نفسه هو 0 و التبــاين

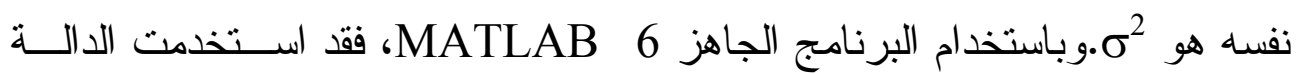

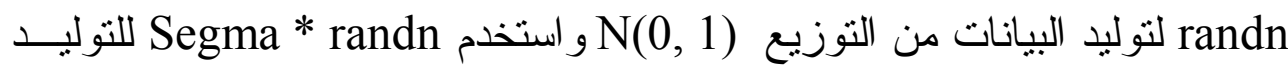

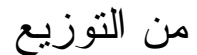

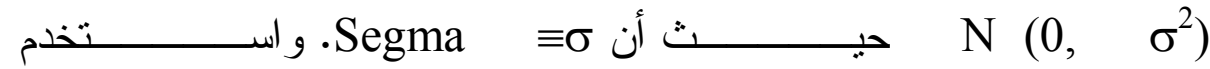
U[ $[-\sqrt{3} \sigma, \sqrt{3} \sigma]$ أهـ $\sqrt{3} \times \operatorname{segma} \times(-1+2 \times$ rand $)$ $\frac{- \text { segma }}{\sqrt{2}} \times \operatorname{Ln}(2 \times$ rand $)$ فقد استخدم المولد الآتي للتوليد منه ، حيث تمت الاستفادة من الخاصية المعروفة في نظرية الاحتمال و التي تتص على

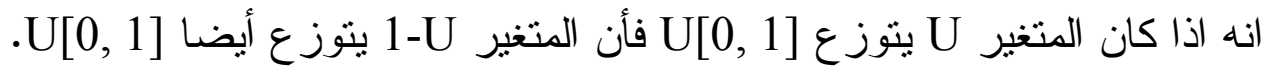

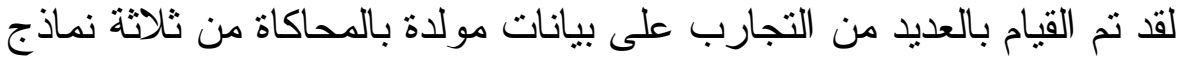

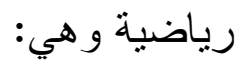


1-نموذج الانحدار الذاتيAutoregressive Model من الرتبة الأولى (1) AR:

$$
\mathrm{X}_{\mathrm{t}}=\mathrm{a} \mathrm{X}_{\mathrm{t}-1}+\mathrm{Z}_{\mathrm{t}}
$$

2-نموذج الأوساط المتحركة Moving Average Model من الرتبـــة الأولـى

$\mathrm{X}_{\mathrm{t}}=\mathrm{b} \mathrm{Z} \mathrm{t}_{\mathrm{t}-1}+\mathrm{Z}_{\mathrm{t}}$

3-نموذج الانحدار الذاتي للاوساط المتحركة

Autoregressive - Moving Average Model :ARMA $(1,1)$

$\mathrm{X}_{\mathrm{t}}=\mathrm{aX} \mathrm{X}_{\mathrm{t}-1}+\mathrm{b} \mathrm{Z} \mathrm{Z}_{\mathrm{t}-1}+\mathrm{Z}_{\mathrm{t}}$

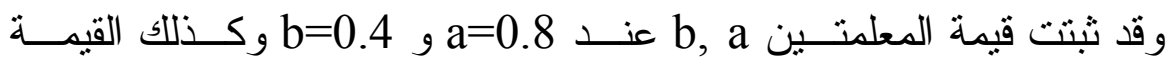

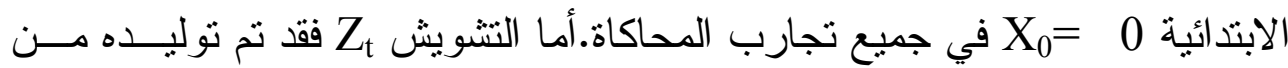
التوزيعات الثناثة - $\sigma^{2}=1,5,25$

لقد تم توليد عينات كل منها بحجم n=500 من كل مــن النمــاذج الثلاثــة

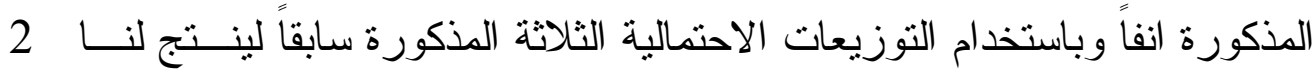

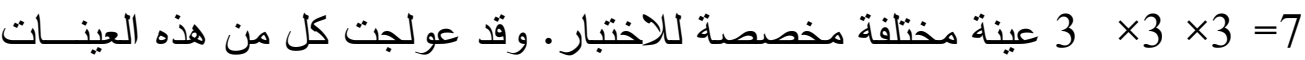

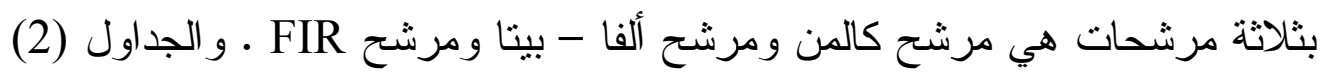

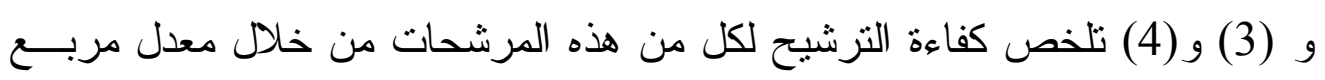
MSE الخطأ

$M S E=\frac{\sum_{t=1}^{n}\left(\hat{X}_{t}-y_{t}\right)^{2}}{n}$

حيث ان yt تمثل المشاهدة المشوشة، تمثل الحالة المرشحة عند الزمن t. 
الجدول (2 ): معدل مربع الخطأ للبيانات المرشحة و المولدة من النموذج (1)

\begin{tabular}{|c|c|c|c|c|}
\hline مرشح FIR & مرشح ألفا- بييًا & Kalman filter & $\begin{array}{c}\text { تباين التثويش } \\
\sigma^{2}\end{array}$ & توزيع التثويش \\
\hline 0.0419 & 0.8186 & 0.0870 & 1 & \multirow{3}{*}{$\mathrm{N}\left(0, \sigma^{2}\right)$} \\
\hline 1.8605 & 23.5076 & 2.9457 & 5 & \\
\hline 24.5729 & 603.8201 & 84.3150 & 25 & \\
\hline 0.0403 & 0.9109 & 0.0838 & 1 & \multirow{3}{*}{$\mathrm{U}[-\sqrt{3} \sigma, \sqrt{3} \sigma$} \\
\hline 4.0944 & 9.0944 & 0.0900 & 5 & \\
\hline 175.7578 & 167.4249 & 0.7381 & 25 & \\
\hline 0.1672 & 0.9577 & 0.1204 & 1 & \multirow{3}{*}{$-\mathrm{L}\left(0, \frac{\sigma}{\sqrt{2}}\right)$} \\
\hline 0.0381 & 0.1098 & 0.1098 & 5 & \\
\hline 0.0449 & 0.9745 & 0.1292 & 25 & \\
\hline
\end{tabular}

الجدول (3 ) معدل مربع الخطأ للبيانات المرشحة والمولدة من النموذج (1)

\begin{tabular}{|c|c|c|c|c|}
\hline مرشح FIR & $\begin{array}{l}\text { مرشع ألفا- بيتا } \\
\alpha-\beta \text { Filter }\end{array}$ & $\begin{array}{c}\text { مرشح كالمن } \text { Kalman filter } \\
\text { Kalman }\end{array}$ & $\begin{array}{c}\text { تباين الثثويش }{ }^{2} \\
\sigma^{2}\end{array}$ & $\begin{array}{c}\text { توزيع التثويش } \\
Z_{t}\end{array}$ \\
\hline 0.0161 & 1.0483 & 0.3713 & $\overline{11}$ & \multirow{3}{*}{$\mathrm{N}\left(0, \sigma^{2}\right)$} \\
\hline 0.4863 & 27.6764 & 11.2049 & 5 & \\
\hline 11.9683 & 795.3262 & 275.7562 & 25 & \\
\hline 0.0181 & 1.1383 & 0.4165 & 1 & \multirow{3}{*}{$U[-\sqrt{3} \sigma, \sqrt{3} \sigma]$} \\
\hline 0.4693 & 10.8595 & 375.5836 & 5 & \\
\hline 16.3010 & 220.6412 & 375.5836 & 25 & \\
\hline 0.0182 & 1.2525 & 0.4204 & 1 & \multirow{3}{*}{$\mathrm{L}\left(0, \frac{\sigma}{\sqrt{2}}\right)$} \\
\hline 0.0172 & 1.0484 & 0.3956 & 5 & \\
\hline 0.0197 & 1.2079 & 0.4534 & 25 & \\
\hline
\end{tabular}


الجدول (4) معدل مربع الخطأ للبيانات المرشحة والمولدة من النموذج

\begin{tabular}{|c|c|c|c|c|}
\hline مرشح FIR & $\begin{array}{l}\text { مرشح ألفا- بيثا } \\
\alpha-\beta \text { Filter }\end{array}$ & $\begin{array}{c}\text { مرشح كالمن } \\
\text { Kalman Filter }\end{array}$ & $\begin{array}{c}\text { تباين الثشويش } \\
\sigma^{2}\end{array}$ & توزيع التثويش \\
\hline 0.0760 & 0.9481 & 0.1947 & 1 & \multirow{3}{*}{$\mathrm{N}\left(0, \sigma^{2}\right)$} \\
\hline 2.6048 & 28.4398 & 6.6685 & $\overline{5}$ & \\
\hline 43.5955 & 717.7972 & 111.6089 & 25 & \\
\hline 0.0723 & 1.0636 & 0.1850 & 1 & \multirow{3}{*}{$U \mid-\sqrt{3} \sigma, \sqrt{3} \sigma}$. \\
\hline 8.1660 & 27.7794 & 20.9057 & 5 & \\
\hline 343.4366 & 965.6822 & 879.2328 & 25 & \\
\hline 0.0749 & 1.1019 & 0.1916 & 1 & \multirow{3}{*}{$\mathrm{L}\left(0, \frac{\sigma}{\sqrt{2}}\right)$} \\
\hline 0.0683 & 1.0111 & 0.1749 & 5 & \\
\hline 0.0807 & 1.1868 & 0.2066 & 25 & \\
\hline
\end{tabular}

ويتضح من الجداول الثلاثة السابقة الكفاءة العالية لمرشح FIR في إعطاء

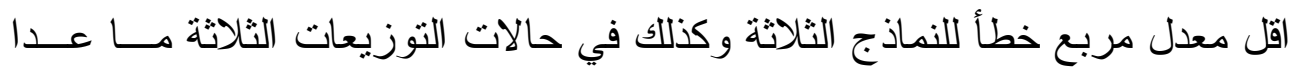

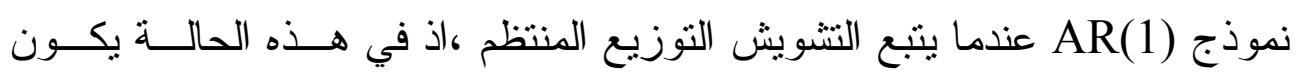

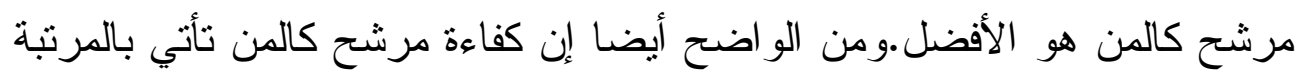
الثانية، أما مرشح ألفا- بيتا فتبدو كفاءته منو اضعة مقارنة مع المرشحين السابقين.

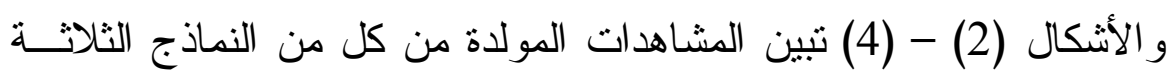

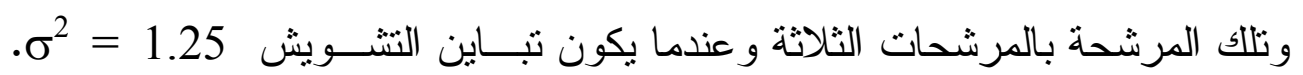

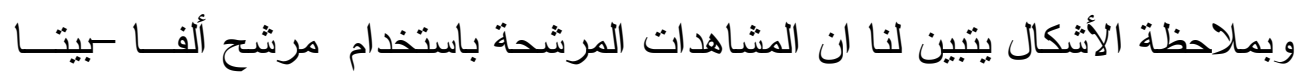
تكون اكثر تشتتاً من تلك التي حصلنا عليها باستخدام كل من مرشح كالمن و

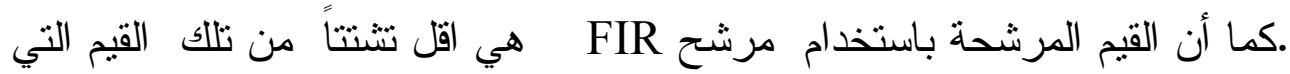


حصلنا عليها باستخدام مرشح كالمن وهذا يفسر النتائج التي تم الحصول عليها من

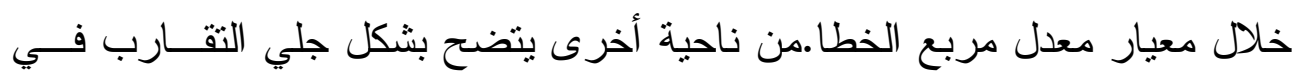

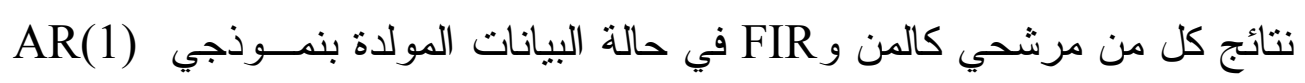
.ARMA $(1,1)$ و

4- - الاستنتاجات و التوصيات:

1- لقد أظهرت نتائج تجربة المحاكاة تواضع نتائج مرشح ألفا-بيتا مقارنة بمرشح FIR ولعل من أسباب ذلك هو ثبوتية ربحية الترشيح لهذا المرشح.

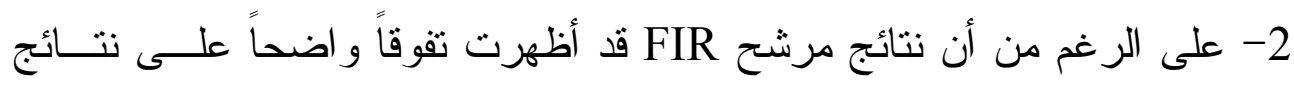

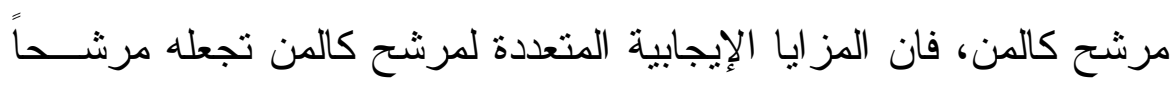

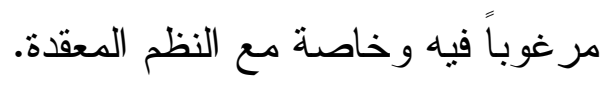

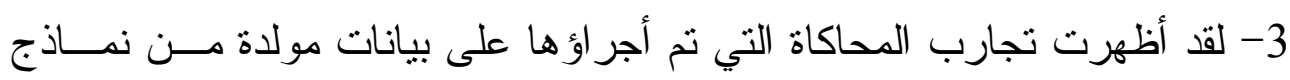

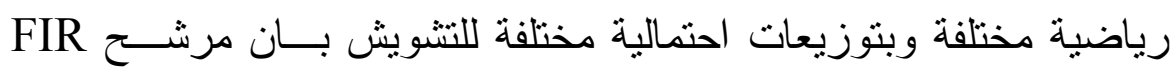

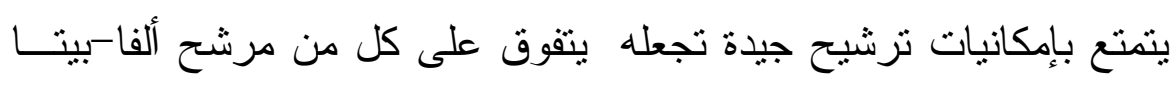
ومرشح كالمن.

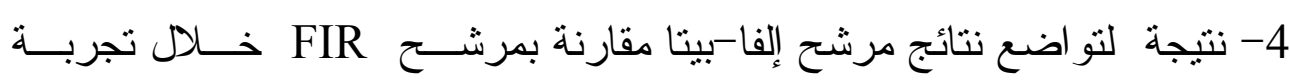

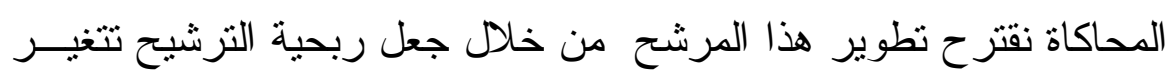

$$
\text { مع الزمن بدلا من ثبوتيتها. }
$$

5- نوصي باستخدام الإصدار ات الحديثة المتعلقة بمرشح كالمن منل مرشح كالمن

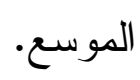




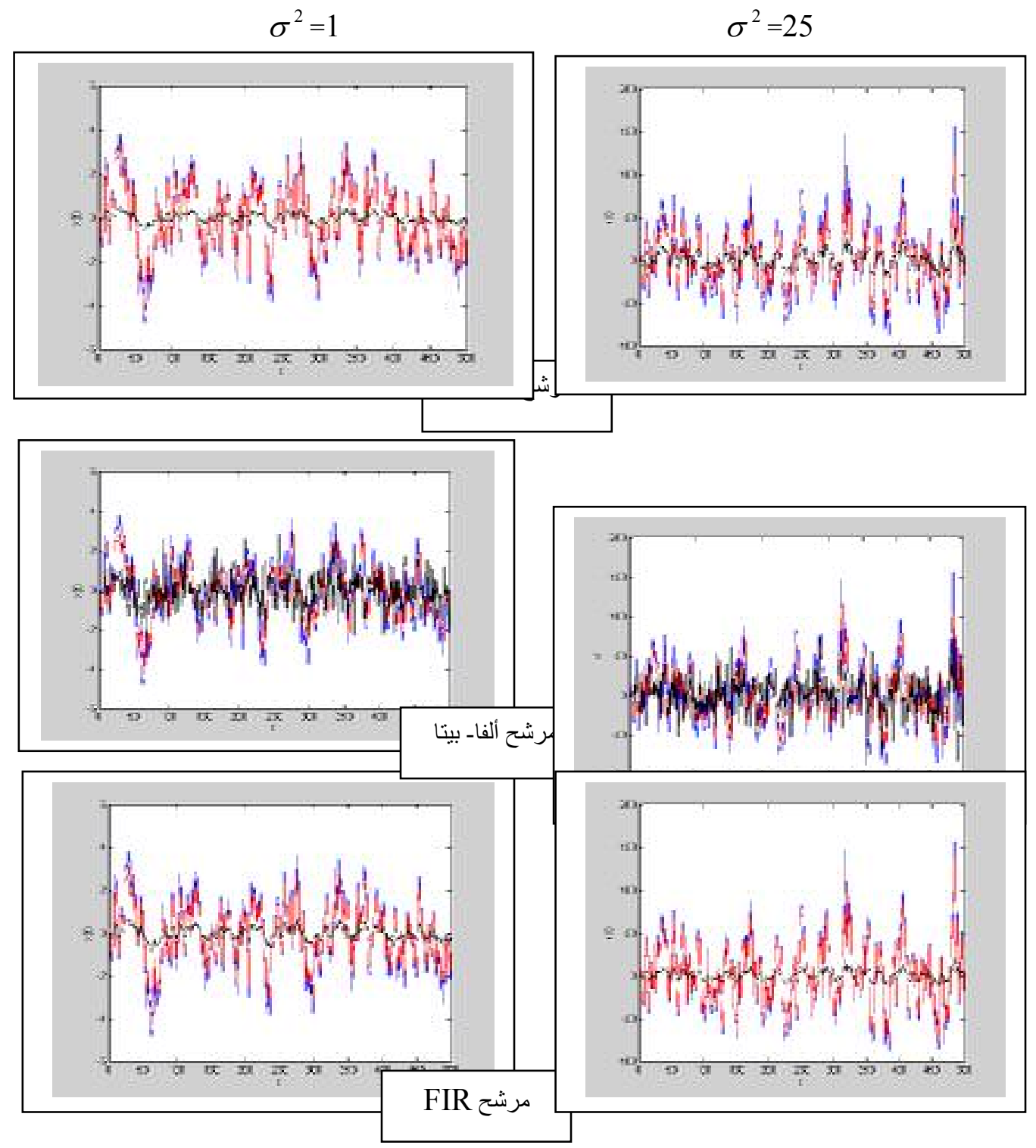

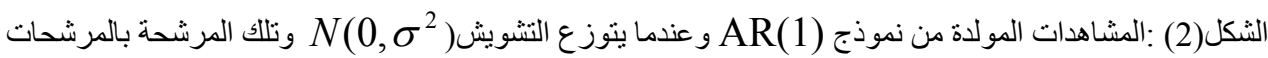
الثلاثة. 

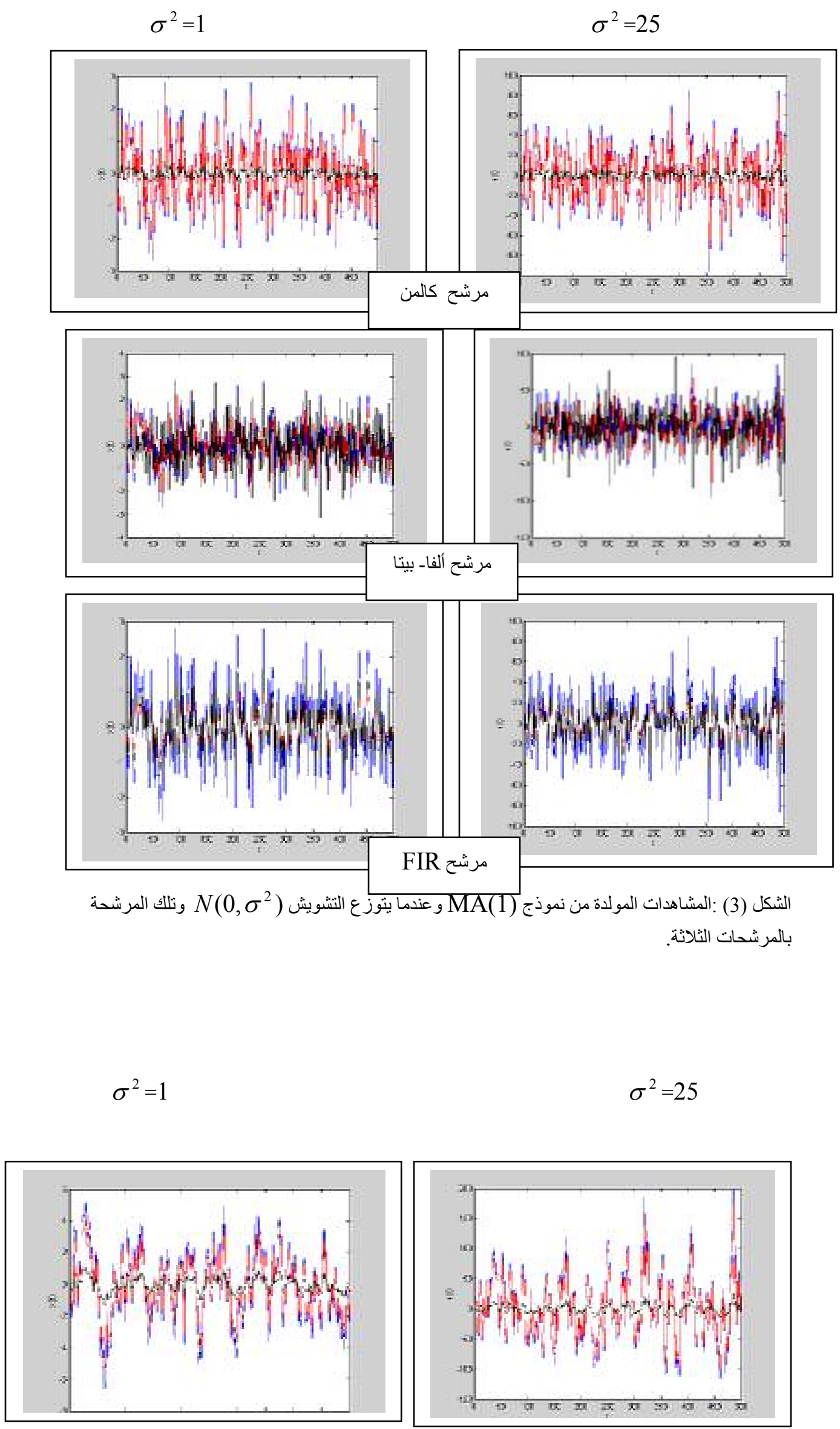


\section{مرشح كالمن}
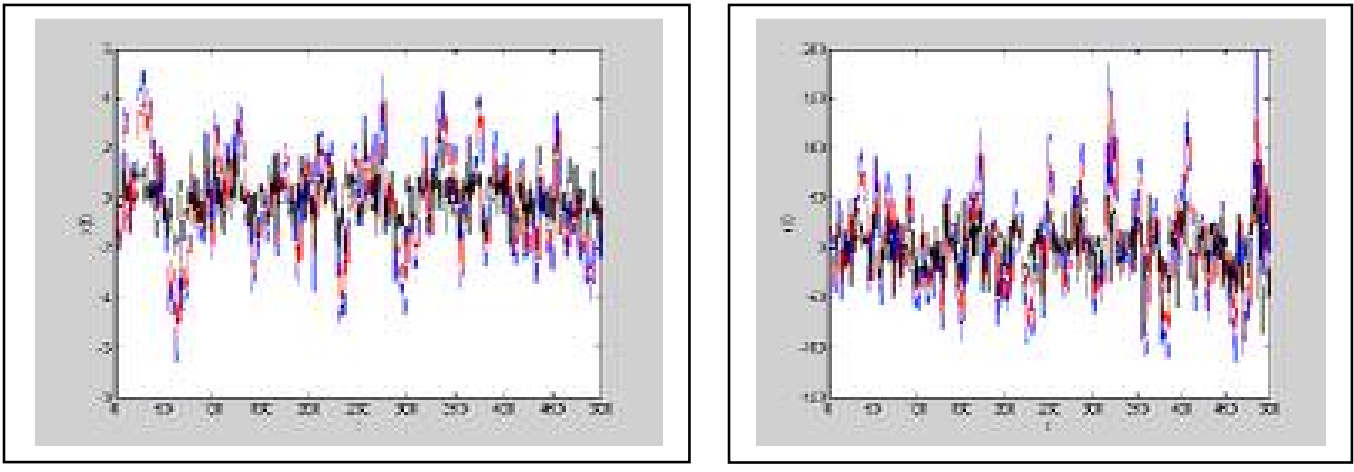

مرشح ألفا- بيتا
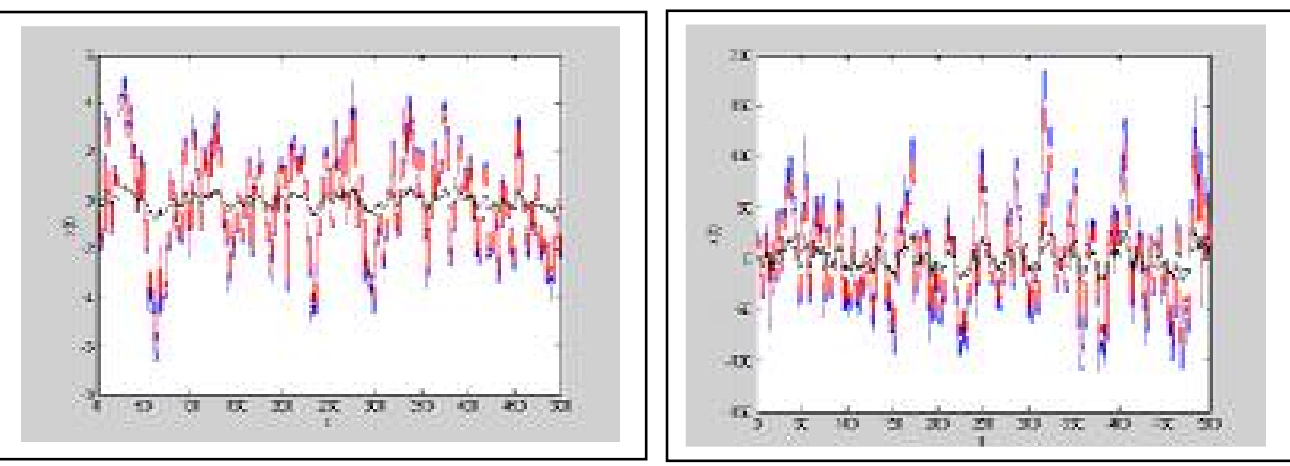

FIR مرشح

الشكل(4) :المشاهدات المولـدة مــن نمــــذج ARMA(1,1 و عنــدما يتــوزع التشويش N(0, $\sigma^{2}$ و تلك المرشحة بالمرشحات الثلاثة. 


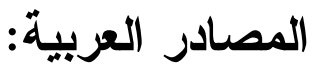

1. 1-الجابري ،عبد الرحمن محمد(2000) ،در اسة لبعض أساليب نرشيح

السلاسل الزمنية مع تطبيقات ـ أطروحة دكتور اه ،غير منشورة،كلية علوم

الحاسبات و الرياضيات، جامعة الموصل المل

2. 2-الحمداني ،مهند سعد الله داؤد(1996) ،مرشح كالمن لبعض النماذج

الحركية الخطية مع المحاكاة ،رسالة ماجستير ،كلية الإدارة و الاقتصــاد ،

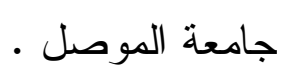

3. 3-الوجيه ،خليل سعيد (2002)، " در اسة عن تعقب الأهداف باستخدام

بعض الأساليب الأكية"، رسالة دكتور اه ، كلية علوم الحاسبات و الرياضيات

$$
\text { جامعة الموصل. }
$$

4. Benedict, T.R. and Brodner, G.W . (1962)." Synthesis of an optimal set of radar track- while - scan smoothing equation”. In IRE Transaction on Automatic Control, volume AC-1

5. Carlson, G.E. (1998). "Singular and linear system Analysis" John Wiley and Sons, NCW York.

6. Kalman,R.E. and Bucy,R.S.(1961).”New results in linear filtering Problems and prediction ".Trans. ASME.J. Basic Eng., Series D,83, 95-108 
7. Kalman,R.E.(1960).”A new approach to linear filtering and prediction problems“. Trans. ASME. J. Basic Eng., Series D, 82,35-45.

8. Kanjilal , p.p.(1995). Adaptive prediction And predictive Control. Peter peregrinusltd . London

9. Kitagawa, G., (1991)." A non-liner smoothing method for time series analysis". Statistic Since. 1: 271-388

10. Sklansky,J.(1957) optimizing the dynamic parameter of attack-while- scan system. RCA Laboratories, Princeton, N.J . 11. Tanazaki, H. (1997),"Nonlinear and nonnormal filters using Monte-Carlo methods". Comp. Stat. And Data Analysis 25, pp.417-439 